This is an Open Access article distributed under the terms of the Creative Commons Attribution-Noncommercial License, which permits unrestricted use, distribution, and reproduction in any noncommercial medium, provided the original work is properly cited.

\title{
POLYCYCLIC AROMATIC HYDROCARBONS WITH SPICA
}

\author{
O. Berné ${ }^{1}$, C. Joblin ${ }^{2,3}$, G. Mulas ${ }^{4}$, A. G. G. M. Tielens ${ }^{5}$, and J. R. Goicoechea ${ }^{1}$ \\ $2{ }^{1}$ Centro de Astrobiología (CSIC/INTA), Madrid, Spain \\ Université de Toulouse ; UPS ; CESR ; 9 ave colonel Roche, F-31028 Toulouse cedex 9, France \\ ${ }^{3}$ CNRS; UMR 5187; 31028 Toulouse, France \\ ${ }^{4}$ INAF Cagliari, Italy \\ ${ }^{5}$ Leiden Observatory, University of Leiden, Leiden, The Netherlands
}

\begin{abstract}
Thanks to high sensitivity, high angular resolution and broad spectral coverage, SPICA will offer a unique opportunity to better characterize the nature of polycyclic aromatic hydrocarbons (PAHs) and very small grains (VSGs), to better use them as probes of astrophysical environments. The angular resolution will enable to probe the chemical frontiers in the evolution process from VSGs to neutral PAHs, to ionized PAHs and to "Grand-PAHs" in photodissotiation regions and HII regions, as a function of $\mathrm{G}_{0} / \mathrm{n}$ (UV radiation field / density). High sensitivity will favor the detection of the far-IR skeletal emission bands of PAHs, which provide specific fingerprints and could lead to the identification of individual PAHs. This overall characterization will allow to use PAH and VSG populations as tracers of physical conditions in spatially resolved protoplanetary disks and nearby galaxies (using mid-IR instruments), and in high redshift galaxies (using the far-IR instrument), thanks to the broad spectral coverage SPICA provides. Based on our previous experience with ISO and Spitzer we discuss how these goals can be reached.
\end{abstract}

Key words: Galaxies: formation - Stars: formation - Missions: SPICA

\section{INTRODUCTION}

The ubiquitous mid-IR emission bands, widely observed in the spectra of dusty astrophysical sources (from protoplanetary disks to starburst galaxies), are attributed to the emission of a family of carbonaceous macromolecules: the polycyclic aromatic hydrocarbons (PAHs). However, because these bands are due to nearest neighbor vibrations of the $\mathrm{C}-\mathrm{C}$ or $\mathrm{C}-\mathrm{H}$ bonds, they are not specific to individual PAH species. Therefore, in spite of their major relevance for astrophysics (as tracers of the presence of UV radiation fields or star forming regions in a broader extragalactic context), the identification of a given $\mathrm{PAH}$ molecule in space has yet not been possible. This contribution explores the new possibilities that could be offered by SPICA spectrometers to better characterize PAHs: In the mid-IR, high angular resolution will enable to better establish the link between the chemical evolution of PAHs and VSGs in connection with the evolution of physical conditions and the formation/excitation of $\mathrm{H}_{2}$. This will then allow to use them as tracers of physical conditions at low and high redshifts. In the far-IR, we expect to possibly detect the low-energy vibrational modes of PAHs which are much more connected to the structure of each molecule and can thus provide an unprecedented characterization of their nature and evolution in space.

\section{Mid-INFRARED OBSERVATIONS OF PAH BANDS}

\subsection{PAHs/VSGS: TRACERS OF PHYSICAL CONDITIONS}

The mid-IR emission of PAHs and VSGs has been well characterized by ISO (see e.g. Peeters et al. 2002; Rapacioli et al. 2005) and more recently by Spitzer (see e.g. Werner et al. 2004; Berné et al. 2007). The observed spectrum usually consists in a set of bands that are most prominent at $3.3,6.2,7.7,8.6,11.3$, and $12.7 \mu \mathrm{m}$. It was established that the modification of the shape of this spectrum can be attributed to alteration of the chemical structure of the emitting component (Peeters et al. 2002; Hony et al. 2001) and that this chemical evolution is strongly connected to the local physical conditions. In particular, models (Tielens 2005) and observations (Joblin et al. 1996; Galliano et al. 2008) have shown that the variations of the 6.2 (or 8.6) to $11.3 \mu \mathrm{m}$ band intensity ratio $\left(I_{6.2} / I_{11.3}\right)$ evolves with the "ionization parameter" $\gamma=G_{0} \times \sqrt{T} / n_{H}$ where $G_{0}$ is the intensity of the UV radiation field in Habing's units, $T$ is the gas temperature and $n_{H}$ the total hydrogen nuclei density. Following this work Berné et al. (2009b) have shown that the combination of the measurement of $I_{6.2} / I_{11.3}$ and of the ratio between the $\mathrm{H}_{2} \quad 0-0 \mathrm{~S}(3)$ and $\mathrm{S}(2)$ line intensities, respectively at 9.7 and $12.3 \mu \mathrm{m}$, allows to derive the individual values of $T$, $G_{0}$ and $n_{H}$ when they fall in the ranges $T=250-1500 \mathrm{~K}$, $n_{H}=10^{4}-10^{6} \mathrm{~cm}^{-3}, G_{0}=10^{3}-10^{5}$ respectively.

\subsection{PAHs/VSGs: ROLE IN THE FORMATION OF $\mathrm{H}_{2}$ (?)}

$\mathrm{H}_{2}$ is the most abundant molecule in the universe but its formation mechanism is still an open question. It is however clear that $\mathrm{H}_{2}$ forms at the surface of grains, as the gas-phase routes are too inefficient under standard ISM conditions (Gould \& Salpeter 1963). The formation rate of $\mathrm{H}_{2}$ in photodissociation regions (PDRs) was found to be larger than that derived from the "classical" formation 
Extracted spectra

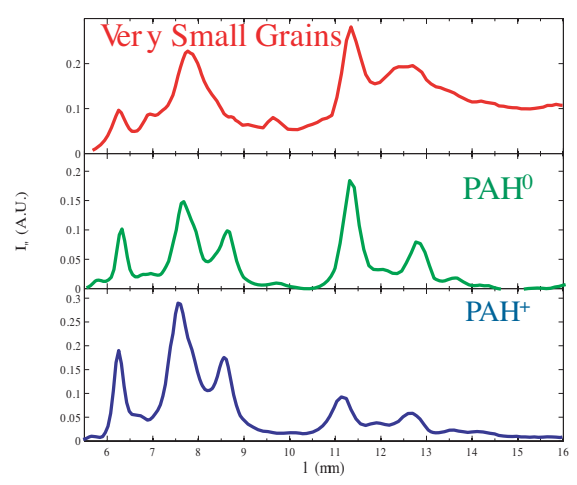

Spatial distribution

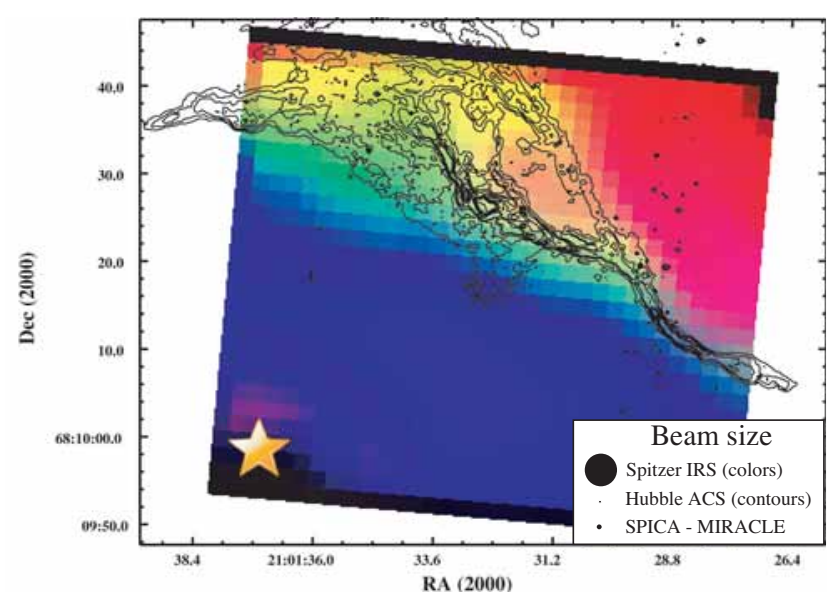

Figure 1. Left: Extracted spectra of VSGs, PAH and PAH $H^{+}$in NGC 7023 N Spitzer cube. Right: Associated distribution maps of the three populations : VSGs in red, $P A H^{0}$ in green and $P A H^{+}$in blue. Colors combine as in an RGB image i.e. green $\left(P A H^{0}\right)+$ red $(V S G s)=y e l l o w$. In contours are shown the filaments detected in ERE with Hubble.

mechanism at the surface of cold grains (Habart et al. 2004). Furthermore, Joblin et al. (2000) have shown that $\mathrm{PAH} / \mathrm{VSG}$ and $\mathrm{H}_{2}$ emissions spatially correlate. In this context, it has been considered that PAHs and/or VSGs could play a role in the catalysis of $\mathrm{H}_{2}$ formation.

\subsection{PAH AND VSG CHEMistry WITH SPICA}

The study of galactic PDRs, where one can resolve the variations of PAH and VSG emissions as the UV field is attenuated, is crucial for the understanding of their chemical evolution and their link with $\mathrm{H}_{2}$ formation. Until now, PDRs have been extensively observed in the mid-IR but only at low angular resolution. There is nevertheless strong evidence that the zone where "everything happens" is in fact very thin and not resolved by Spitzer and ISO. Indeed, the evolution from VSGs to PAHs occurs in a region of less than 2 magnitudes where physical conditions vary significantly. Considering that the molecular cloud has a density of $10^{5} \mathrm{~cm}^{-3}$ this extinction represents, at a distance of $500 \mathrm{pc}$, an angular scale of $\sim 1.3$ " requiring subarcsecond resolution to be probed. Observational evidences of this sharp variation of density and radiation field are numerous: Berné et al. (2008) have shown that the transition from VSGs to PAHs, traced by the Extended Red Emission (ERE), is very sharp (filaments of $\sim 1$ ") in NGC 7023 (Fig. 1). The same conclusions are found for NGC 2023 (Pilleri et al. in prep.). $\mathrm{H}_{2} 2.12 \mu \mathrm{m}$ high angular resolution data also evidence this very thin transition (Lemaire et al. 1999). Radio interferometric (Gerin et al. 2009), and near-IR $\mathrm{H}_{2}$ (Habart et al. 2005), observations have evidenced the arcsecond scale chemical stratification in the Horshead PDR. In the same region, a steep density gradient has been put forward by Pety et al. (2005) as well as a possible destruction of PAH molecules occurring within these small spatial scales. In Monoceros R2 and the Orion bar, $\mathrm{H}_{2} 2.12 \mu \mathrm{m}$ observations also suggest the presence of a thin membrane separating the molecular gas from the HII region (Walmsley et al. 2000). Unfortunately, it has for now been impossible to spatially resolve this frontier with spectral maps in the mid-IR as the best achieved angular resolution with Spitzer IRS was 3.6". This prevents from understanding the link between the chemical evolution of PAHs and VSGs and the origin of the ERE or $\mathrm{H}_{2}$ formation processes. The MIRACLE camera onboard SPICA will be particularly suited to solve this observational issue. With a subarcsecond resolution, while having a low spectral resolution adapted to PAH bands, MIRACLE will enable, for the first time, to probe these chemical frontiers that are crucial for our understanding of PAHs/VSGs and $\mathrm{H}_{2}$ photochemistry (Fig. 1). One key point is the use of the imaging with band filters at $\mathrm{R} \sim 5$. The fitting of the limited number of spectral points with a linear combination of $\mathrm{PAH}^{0}, \mathrm{PAH}^{+}$and VSG spectra (see templates adapted from Fig. 1 in Berné et al. 2009) will provide, for each position in the images, a good estimation of the full mid-IR spectra as well as the spatial distribution of PAH and VSG populations, without spending any time doing spectroscopy. This can only be achieved if the number of filters in the $5-14 \mu \mathrm{m}$ range is sufficient $(>10)$. Furthermore, the large field of view (FoV) provided by SPICA in the mid-IR will allow to map much larger regions at once. For instance, the NGC 7023 reflection nebula fits entirely in the MIRACLE FoV (i.e. North, East and South PDRs at the same time). 

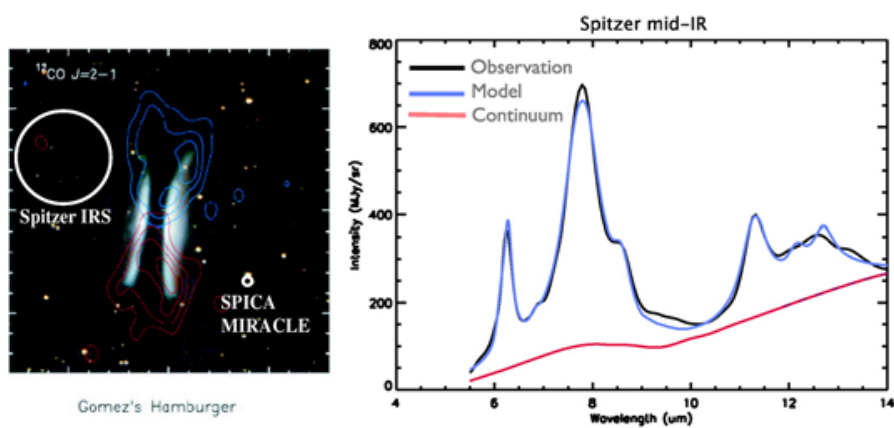

Figure 2. Left: Hubble Space telescope observations of Gomez's Hamburger in the visible. Overlaid in colors the ${ }^{12} \mathrm{CO} \mathrm{J}=2-1$ emissions for velocities between 0.9 and $4.1 \mathrm{~km} \mathrm{~s}^{-1}$, Doppler shifted towards blue (north) and red (south) from Bujarrabal et al. (2009). White circles are the beams of Spitzer IRS and SPICA. Right: The Spitzer-InfraRed Spectrograph spectrum of Gomez's Hamburger in black and adjusted PAH/VSG model (from Berné et al 2009) in blue.

\section{PAHS IN PROTOPLANETARY DISKS}

It has become clear, in the recent years, that the disks of gas and dust that form around young stellar objects are the cradles of planetary formation (see e.g. recent observations of exoplanet embedded in the disk of Formalhaut by Kalas et al. 2008). PAHs and VSGs are know to be present in the gas-rich protoplanetary disks and play multiple roles: (i) they can be considered as building blocks from which larger bodies can form by aggregation, (ii) because they are a major source of optical thickness in the UV (Draine \& Li 2007) they shield the gas from photodissociation (Dullemond et al. 2007; Berné et al. 2009) and slow the photo-evaporation process (Alexander 2008). Only very few studies from the ground have enabled to look at the properties of PAHs within protoplanetary disks, and only in imaging through broad-band filters (Lagage et al. 2006; Geers et al. 2007). MIRACLE will spatially resolve such disks (see e.g. Fig. 3), and with imaging at $\mathrm{R} \sim 5$ provide the maps of $\mathrm{PAHs}^{\mathrm{O} /+}$ and VSGs. Using the methods described in Sect.2.1 we will be able to obtain the physical properties of different regions of the surface at the disk.

\section{REDSHIFTED MID-IR PAH BANDS}

Spitzer has brought clear evidence that PAH bands are present in the emission of galaxies dominated by star formation at $z>2$ (see e.g. Pope et al. 2008). Star formation rates (SFR) of galaxies can then be estimated by relating the PAH luminosity to the total IR luminosity (see e.g. Brandl et al. 2006) and then the total IR luminosity to the SFR using the Kennicutt (1998) law. Recent studies have also intended to relate the ionization fraction / size of PAHs to the SFR (O'Dowd et al. 2009). SPICA will enable to better characterize the relationship between SFR and $\mathrm{PAH}$ emission, and will provide $\mathrm{PAH}$ spectra of galaxies at higher red-shifts (see cosmological implications in the extragalactic section of these proceedings). One important point is that with it's unprecedented sensitivity, SPICA will allow to observe both the $\mathbf{P A H}$ and $\mathbf{H}_{2}$ emissions at high $z$. As show by Berné et al. (2009b) this can be very useful to learn about the physical conditions prevailing in the emitting environment.

\section{FAR-IR MODES OF PAHS}

Far-IR emission bands of PAHs would provide specific fingerprints of individual molecules. These low-energy vibrations involve the bending of the whole PAH skeleton (mostly out-of-plane) and are thus intrinsically related to the structure of each possible PAH carrier. Unfortunately, PAHs tend to release their vibrational energy mostly through mid-IR emission, and thus their far-IR bands are expected to be very weak. As an example, $0.2 \%$ of the total UV energy absorbed by a PAH like coronene, will be emitted in FIR band emission (Joblin et al. 2002). Therefore, the PAH FIR band emission is expected to be difficult to detect in space. Nonetheless, it can be shown that if all the mid-IR emission observed in the ISM was due to only a few large PAH molecules, their far-IR emission band should be detectable even with a low $(<100)$ signal-to-noise ratio (see Mulas et al. 2006 and Fig. 3). ISO/LWS detected several unidentified far-IR bands (Cernicharo et al. 2002; Goicoechea et al. 2004) although none could specifically be attributed to PAH emission. The non detection of such bands with ISO combined with other evidence in the midIR (Peeters et al. 2002), suggest that a scheme in which there are only a few different PAH molecules is unlikely. If instead, there are 50 different PAHs responsible for the mid-IR band spectrum, the strongest band emitted by all of them in the far-IR will have a peak intensity of $\sim 1 \%$ of the thermal continuum intensity. While this emission may seem extremely weak, it is not impossible to detect it, given the progresses made in far-IR space instruments, and the prospects of much improved sensitivity with the SPICA/SAFARI spectrometer. If there were really 50 different PAHs in space, the problem might come from somewhere else: the relatively uncertain wavelength position of these PAH bands, and from spectral confusion. Indeed, the more numerous interstellar PAHs are, the more numerous far-IR PAH bands there will be. Fortunately, recent progress in the field of interstellar PAHs suggests that there are in fact only a limited number of large and compact PAHs in space (see Tielens 2008 for review), that can resist the harsh interstellar conditions thanks to their ability to redistribute the energy they absorb efficiently inside the molecule, these are the so called Grand-PAHs. The instantaneous broad band coverage of SAFARI-FTS will be specially adapted for deep searches of far-IR bands where the wavelength positions are not constrained spectroscopically and can appear in the whole domain. The discovery of the specific PAH carriers responsible of the 

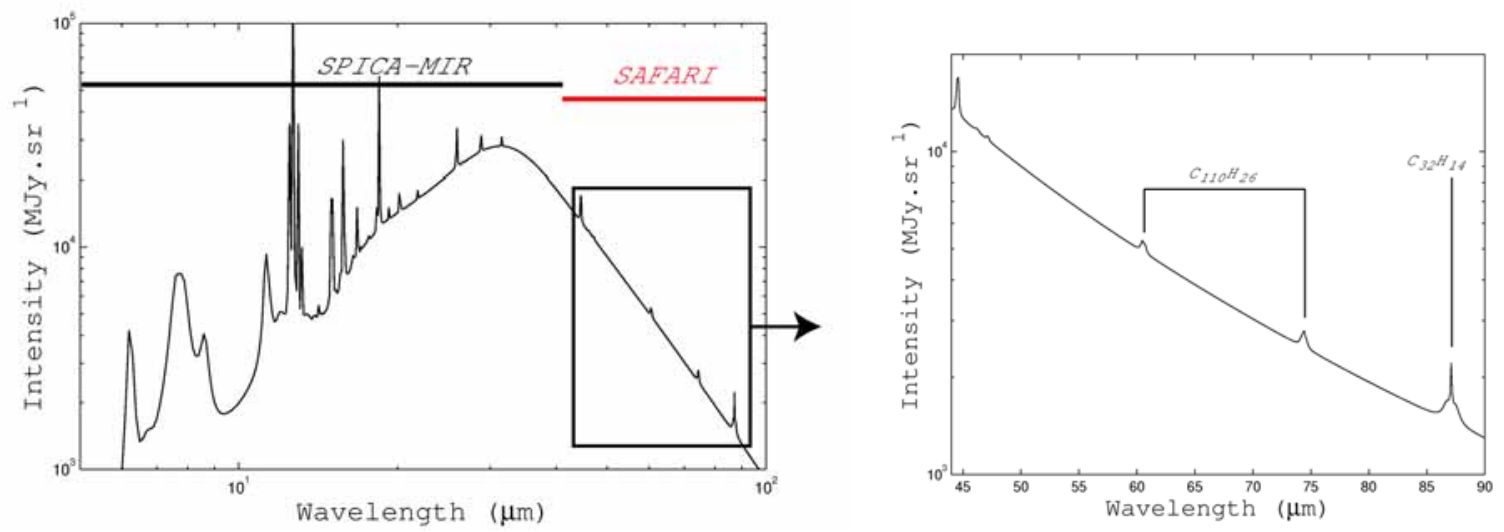

Figure 3. Modeled spectrum of a PDR with a $G_{0}=10^{5}$ radiation field, column density of $N_{H}=10^{20} \mathrm{~cm}^{-2}$ and $20 \%$ of carbon locked in PAHs and assuming that the mid-IR bands are due to $C_{110} H_{23}$ and $C_{32} H_{14}$. The adopted spectral resolution is $\sim$ 1000. The mid-IR PAH bands in the spectrum and the continuum emission calculated with the model of Draine $85 \mathrm{Li}$ (2007). The far-IR emission PAH bands are calculated using the photochemical model of Mulas et al. (2006) using IR cross sections calculated by Malloci et al. (2007) and I. Cami (private com.).

widespread mid-IR PAH emission (local and extragalactic) will constitute a tremendous step forward for our understanding of the chemical complexity of the universe. Before that, unprecedented efforts in parallel instrumental, theoretical, laboratory and observational aspects will have to be carried out. Herschel will be limited in terms of wavelength coverage and sensitivity in the far-IR, and thus SPICA/SAFARI can represent our first chance to detect specific PAHs.

\section{ACKNOWLEDGEMENTS}

OB is supported by JAE-Doc CSIC fellowship. JRG was supported by a Ramon y Cajal research contract from the spanish MICINN and co-financed by the European Social Fund. OB, and CJ acknowledge the french national program PCMI.

\section{REFERENCES}

Alexander 2008 NewAR 52, 60

Berné, et al. 2009, ApJL, accepted

Berné, et al. A\&A, 495, 827

Berné, O. et al. 2008, A\&A, 479, 41

Berné, O. et al. 2007, A\&A, 469, 575

Brandl et al. 2006, ApJ, 653, 1129

Bujarrabal et al. 2009 A\&A, 500, 1077

Cernicharo, J et al. 2002, ApJ, 580, L157

Draine, B. T., Li, A. 2007, ApJ, 657, 810

Dullemond et al. 2007 A\&A, 473, 457

Galliano, F. et al. 2008, ApJ, 679, 310

Geers et al. 2007 A\&A, 469L, 35

Gerin, M. et al. 2009, A\&A, 494, 977

Goicoechea, J. R., et al. 2004, ApJ, 609, 225

Gould \& Salpeter, 1963, ApJ, 138, 393

Habart, E. et al. 2005, A\&A, 437, 177

Habart et al. 2004, A\&A, 414, 531

Hony, S. et al. 2001, A\&A, 370, 1030

Joblin, C. et al. 2008, A\&A, 490, 189
Joblin, C. et al. MolPhys, 100, 22, 3595

Joblin, C. et al. 2000, "Molecular hydrogen in space", Cam. Univ. Press, 2001. xix, 326

Joblin, C. et al. 1996, ApJ, 460, L119

Kalas et al. 2008 Science 322, 1345

Kennicutt, R. C., Jr. 1998, ARA\&A, 36, 189

Lagage P. et al. 2006, Science, 314, 621

Lemaire et al. 1999, A\&A, 349, 253

Malloci et al. 2007, ChemPhys, 332, 353

Mulas, G. et al. 2006, A\&A, 460, 93

O'Dowd, M. J. 2009, arXiv0909.2279O

Peeters. E. et al. 2002, A\&A, 390, 189

Pety, J. et al. 2005, A\&A, 435, 885

Pope, A. et al. 2008, ApJ, 675, 1171

Rapacioli, M. et al. 2005, A\&A, 429,193

Tielens 2008 ARA\&A,46,289

Tielens, A. 2005 Cambridge University Press

Tielens, A. et al. 1993, Science,262, 86-89

Werner, M. W. et al. 2004, ApJS, 154, 309 